\title{
ДИАГНОСТИРОВАНИЕ СОСТОЯНИЯ ГАЗОПЕРЕКАЧИВАЮЩИХ АГРЕГАТОВ СПЕЦИАЛЬНЫМИ МЕТОДАМИ ИНТЕРПРЕТАЦИИ СПЕКТРОВ ВИБРОСКОРОСТИ
}

\author{
Байков Игорь Равильевич', \\ pte@rusoil.net
}

\author{
Смородова Ольга Викторовна', \\ olga_smorodova@mail.ru
}

\author{
Китаев Сергей Владимирович', \\ svkitaev@mail.ru
}

\author{
Шаммазов Айрат Мингазович', \\ pte@rusoil.net
Уфимский государственный нефтяной технический университет, Россия, 450064, г. Уфа, ул. Космонавтов, 1.

\begin{abstract}
Актуальность исследования обусловлена необходимостью разработки дополнительных методов оценкитехнического состояния газоперекачивающих агрегатов компрессорных станций магистральных газопроводов. Аварийные ситуации на компрессорных станцияхвлекут за собой последствия глобального характера для материальной базы и окружающей среды. Основным направлением обеспечения безаварийной работы компрессорных станций является поддержка технического состояния газоперекачивающих агрегатов на требуемом уровне.

Цель: разработка метода оценки технического состояния газоперекачивающих агрегатов интерпретацией виброспектра колебаний корпуса подшипника турбины низкого давления агрегата с формулировкой заключения «есть дефект/нет дефекта».

Объекты: газоперекачивающие агрегаты ГТК-10, эксплуатируемые в ПАО «Газпром». Агрегаты данного типа обеспечиваютболее 8 ГВт установленной мощности, что составляет более 20 \% в газотранспортной системе страны. Базой данных для проведения исследований является система данных результатов вибродиагностики агрегатов за 4 года.

Методы. Для повышения достоверности интерпретации спектров колебаний подшипниковых узловпредлагается использовать метод построения разделяющей поверхности в 340-мерном фазовом пространстве по характеристикам обучающей выборки виброспектров. Установлено, что достоверность определения состояния агрегата при этом связана прямо пропорционально с количеством спектров в обучающей выборке, сформированной на основе информации по вибрационным обследованиям. Максимальная адекватность выводов при идентификации состояния агрегатов имеет место при описании вибросигнала преобразованием Фурье с ярко выраженными амплитудами виброскорости.

Результаты. Построена гиперплоскость для определения уровнятехнического состояния газовых турбинкомпрессорных станций ПАО «Газпром» интерпретацией спектров виброобследования. Учитывая уменьшение обучающей выборки при отсеивании части спектров, наиболее препятствующих реализации алгоритма, ошибка при распознавании текущего вибросигнала агрегата прогнозируется в пределах 15-30\%.
\end{abstract}

\section{Ключевые слова:}

Обучающая выборка, виброскорость, разделяющая поверхность, размерность, алгоритм, таксон.

\section{Введение}

Стратегические планы газовой промышленности, во многом определяющей техногенное развитие страны, определяются состоянием и условиями эксплуатации перекачивающего оборудования магистральных газопроводов [1]. В связи с этим разработка способов достоверного диагностирования технического состояния агрегатов [2] характеризуется не снижающимся интересом как для дочерних предприятий, так и для всей газотранспортной системы [3].Одной из причин повышенного внимания к диагностике вообще и вибродиагностике в частности $[4,5]$ является требование сохранения работоспособности газоперекачивающего оборудования при развитии процесса старения и деградации.

Общепринятый подход к идентификации состояния газоперекачивающих агрегатов (ГПА) с помощью виброспектров колебаний зачастую не позволяет адекватно оценить техническое состоя- ние агрегатов [6]. При этом основным препятствием является неоднозначность спектральной картины развития дефектных разрушений [7]. Для повышения достоверности интерпретации спектра виброскорости в статье предлагается алгоритм, разработанныйс использованием концепции распознавания образов [8].

\section{Интерпретация виброспектров методами} теории распознавания образов

В традиционной постановке задача распознавания образов сводится к идентификации типа объекта по его качественным или количественным признакам [9]. В таких задачах предполагается, что для настройки алгоритма имеется обучающая выборка [10], то есть некоторая совокупность спектров виброскорости, классификация которых достоверно априори известна («исправный/дефектный»). При этом для достоверной классификации рабочего виброспектра обучение алгоритма дол- 
жно быть реализовано обучающей выборкой, достоверно интерпретирующей состояние агрегата в условиях, идентичных условиям текущей эксплуатации оборудования [11]. Принимая во внимание незначительную аварийность газоперекачивающих агрегатов, естественным образом встает вопрос минимального объема обучающей выборки. Достаточно частым случаем является ситуация, когда объем обучающей выборки значительно меньше количества диагностических признаков. В этом случае процедура построения линейного решающего правила [12] является оптимальным решением.

К признакам тестирования частотных рядов амплитуд виброскорости предъявляется ряд требований:

- простота их экспериментальногоконтроля;

- доступность и несложностьколичественной оценки;

- взаимнаянезависимость [13].

Кроме того, повышение достоверности процедуры реализации алгоритма достигается наращиванием базы определяющих признаков [14].

При подготовке к реализации процедуры оценки технического состояния ГПА в качестве определяющих критериевбыла рассмотрена совокупность различных параметров виброспектров колебаний подшипниковых узлов турбин низкого давления ГПА:

- частотный ряд амплитуд виброскорости;

- амплитуды виброскорости на кратных частотах спектра при узкополосном частотном анализе;

- СКЗ виброскорости по всему спектру и по частотным полосам;

- спектральная плотность по всему спектру и в частотных диапазонахпредполагаемых дефектов ГПА.

Для отбора наиболее информативных показателей был определен уровень значимости каждого из них [15]. В результате в качестве оптимального признака диагностирования технического состояния агрегатов определен частотный ряд 340 aмплитуд виброскорости колебаний опорно-упорного подшипника агрегата [16].

Такой подход отображает каждый виброспектр из 340 амплитуд частотного ряда в системе координат 340-мерного пространства отдельной точкой [17]. Оптимальное формирование базы признаков интерпретации вибрспектров приводит к дифференциации совокупностей образов «исправных/дефектных" агрегатов в 340-мерной системе координат [18]. Полученные таксоны могут быть разделены в пространстве геометрически, аналитический образ разделяющей геометрической формы может быть установлен в рамках итерационного процесса [19].

После формирования базы показателей интерпретации виброспектров не менее важным моментом является подбор элементов обучающей выборки. В нее должны войти спектры, зарегистрированные при однозначно дефектном и однозначно исправном состоянии ГПА (группа 2 и группа 1 соответственно). Опыт эксплуатации и ремонта ГПА газотранспортной системы показывает, что для многих агрегатов (в частности, ГТК-10) максимальное развитие дефекта в роторной конструкции достигается за период не более 1 месяца. Продолжение эксплуатации оборудования при этом приводит либо к аварийному отказу, либо агрегат выводится в ремонт [19].

Таким образом, при анализе ретроспективной базы данных вибродиагностики агрегатов марки ГТК-10 были использованы следующие временные диапазоны дифференциации:

- группа 1 - регистрация спектра более 2 месяцев до аварии;

- группа 2 - регистрация спектра менее 1 месяца до аварии.

В результате временного анализа базы данных вибродиагностики была сформирована обучающая выборка виброспектров. По каждому направлению возможных колебаний - вертикальному, горизонтальному, осевому - длина обучающей выборки составила около 35 спектральных сигналов. Таким образом, при значительном, практически десятикратном отличии размерности диагностических признаков от количества спектров в обучающей выборке оптимальным считается разделение таксонов групп спектров посредством гиперплоскости [8].

Задача состоит в идентификации такого направления $\psi_{0}$ в 340-мерном пространстве признаков, чтобы расстояние между проекциями таксонов 1 и 2 группы на это направление было максимальным. Линия геометрического разделения оболочек таксонов формируется по нормали к направлению $\psi_{0}$, через центральную точку отрезка между областями спектров заведомо дефектного и заведомо исправного состояния ГПА (рис. 1). Априори полагают, что длина отрезка между таксонами вдоль оси $\psi_{0}$ превышает предложенный параметр $\rho$.

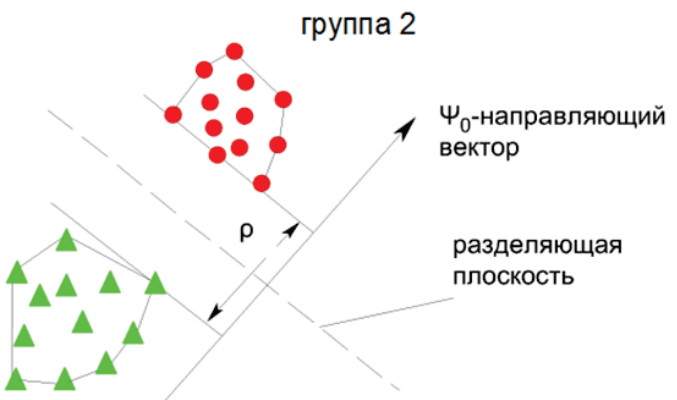

группа 1

$$
0
$$

Pис. 1. Таксоны компонентов в процессе обучения алгоритма в виде проекции 340-мерного пространства амплитуд виброскорости

Fig. 1. Taxons of components in algorithm learning in the form of a projection of the 340-dimensional space of vibration velocity amplitudes 
Процедура построения геометрической поверхности раздела таксонов предваряется отсевом спектров виброскорости по аналогии с грубыми погрешностями измерений. Показателем необходимости отсева какого-либо спектра является его препятствие четкому разделению групп спектров. Необходимо учитывать, что уменьшение объема обучающей выборки за счет отсеивания части ее элементов приводит к снижению достоверности тестирования рабочих спектров в будущем на основе положения их в фазовом пространстве в виде точки относительно построенной разделяющей плоскости.

Следует подчеркнуть, что именно качество обучающей выборки и ее объем определяют уровень достоверности заключения о механическом состоянии агрегата по результатам вибрационной диагностики ГПА [20]. Последовательность действий при построении разделяющей плоскости между таксонами облучающей выборки представляет собой следующий ряд.

Для наполнения обучающей выборки были подобраны вибросигналы за последние 4 года эксплуатации газоперекачивающих агрегатов в однозначно исправном и однозначно предаварийном состоянии:

- 120 сигналов колебаний подшипниковых узлов турбины низкого давления в горизонтальном, вертикальном и осевом направлениях колебаний (отсутствие дефекта);

- 50 сигналов колебаний подшипниковых узлов турбины низкого давления в горизонтальном, вертикальном и осевом направлениях колебаний (предаварийное состояние).

Максимальная частота в спектрах составляла 400 Гц с частотой дискретизации на уровне 1,15 Гц.

Примем обозначения: $B_{i}(i=1 . . .40)$ - для спектров однозначно исправного состояния агрегатов; $B_{i}(j=1 . . .10)$ - для спектров в предаварийном состоянии агрегатов.

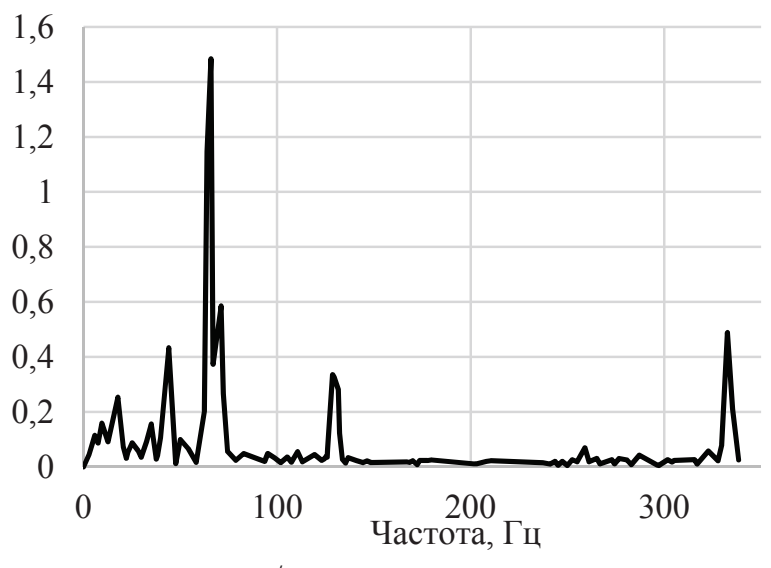

$a-\mathrm{Mм} / \mathrm{c}$, до нормирования
Каждому спектру в этих группах соответствует точка в 340-мерном пространстве диагностических признаков, которыми являются амплитуды частотных рядов Фурье-разложения вибросигнала. Для обоснованного сопоставления амплитуд разных спектров на одной частоте (рис. $2, a$ ) было проведено намеренное выравнивание их уровней значимости нормированием:

$$
A_{k m}=\frac{B_{k}-\min B_{m}}{\max B_{m}-\min B_{m}} \times 255,
$$

где $\min (\max ) B_{m}=\min (\max )\left(B_{m i}, B_{m j}\right)-$ наименьшее (наибольшее) значение виброскорости при $(m)$ Гц по двум таксонам элементов; 255 - нормирующий множитель для обеспечения наибольшей точности при оцифровке вибросигналов диагностической базы.

Спектр вибрации, получаемый в результате данной нормировки, представлен на рис. 2 , б.

В результате обработки диагностической базы данных было установлено, что на любой частоте спектра с одинаковой вероятностью может иметь место амплитуда сигналапроизвольной величины в интервале $(0 ;+1)$. Это позволяет обеспечить равный уровень значимости для каждой частоты виброспектра.

В соответствии с поставленной задачей, процедура обучения алгоритма сводится к нахождению аналитического уравнения плоскости

$$
\sum_{m=1}^{340} \alpha_{m} A_{m}=0
$$

которая позволит дифференцировать таксоны группы 1 и группы 2 так, что интегральное расстояние от плоскости до каждого элементатаксонов будет наибольшим из всех возможных.

На нулевой итерации $(k=0)$ принимают все компоненты вектора $\alpha_{m}$ предельно малыми, незначительно больше нуля- $\alpha_{m}=0,001$. Последующая реализации алгоритма показала состоятельность такого задания компонентов для нахождения аналитического уравнения гиперплоскости.

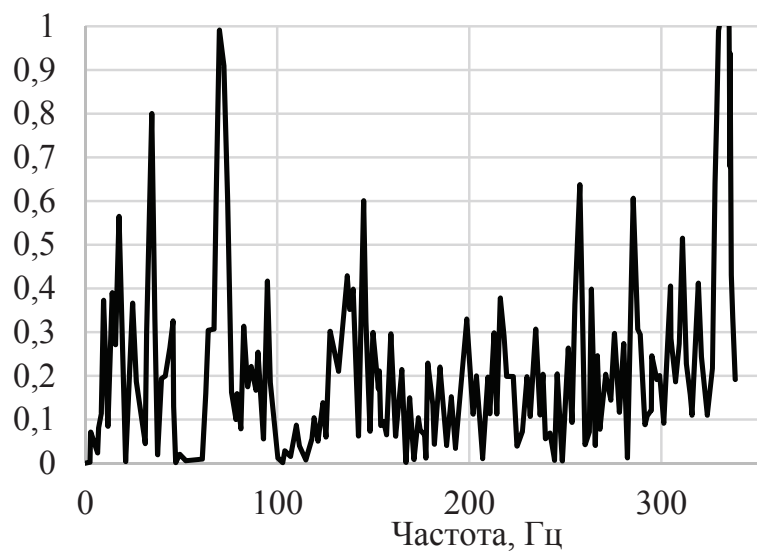

б - отн. ед., после нормирования

Pис. 2. Норлирование спектра виброскорости по величине алплитуды

Fig. 2. Normalization of vibration velocity spectrum by the amplitude magnitude 
Компоненты вектора разделяющей плоскости $\psi_{0}$ были определены как разность между расстояниями каждого элемента группы 1 и группы 2 до нее соответственно [9]:

$$
\psi_{m}^{k}=\sum_{i=1}^{40} \sum_{j=1}^{10} \alpha_{m}^{k}\left(A_{i m}-\bar{A}_{j m}\right) .
$$

Для компонентов направляющего вектора $\alpha_{m}$ в первой итерации будет справедливо соотношение

$$
\alpha_{m}^{1}=\alpha_{m}^{0}+h \frac{\psi_{m}^{0}}{\sqrt{\sum_{m=1}^{340}\left(\alpha_{m}^{0}\right)^{2}}},
$$

где постоянная $h$ принимается из условия обеспечения относительно малого приращения компонентов $\alpha_{m}^{0}$.

Закономерной при практической реализации алгоритма является оценка достаточного для дифференциации таксонов спектров обучающей выборки количества итераций. Специфика процедуры такова, что при увеличении количества итераций наиболее значимые частоты спектров по уровню значимости все более доминируют над остальными. При малом количестве итераций - после первой итерации (рис. $3, a$ ) структура спектра меняется незначительно и практически соответствует началу процесса (рис. 2, б). Уже после третьей итерации (рис. 3 , б) значимость диапазона в окрестности частоты первой роторной гармоники становится критически преобладающей.

Одновременно практически все амплитуды виброскорости в остальной части спектра были сглажены, и, следовательно, информация обо всех остальных структурных особенностях спектра утеряна. В результате авторами в качестве оптимальной была выбрана процедура оценки коэффициентов $\alpha_{m}$ разделяющей гиперплоскости в последовательности трех шагов.

Для диагностирования текущего механического состояния газоперекачивающих агрегатов по результатам виброобследования рекомендуется

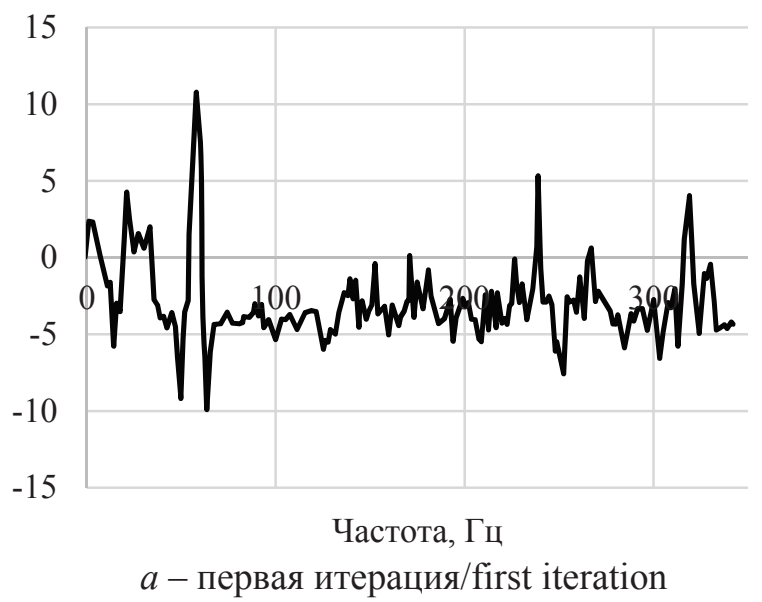

Pис. 3. Уровень значимости частот спектра

Fig. 3. Significance level of the spectrum frequencies определять расстояние от соответствующей точки в 340-мерном пространстве амплитуд виброскорости до разделяющей плоскости по формуле:

$$
L=\frac{\sqrt{\sum_{m=1}^{340} \alpha_{m} A_{m}}}{\sqrt{\sum_{m=1}^{340} \alpha_{m}^{z}}} .
$$

\section{Результаты интерпретации спектров виброскорости}

По результатам вибрационного обследования газоперекачивающих агрегатов было проведено обучение алгоритма, построение разделяющей гиперплоскости и установлено положение точек таксонов (группа 1 и группа 2) относительно построенной плоскости в двумерном пространстве (рис. 4).

Двумерная проекция идентифицированной разделяющей плоской поверхностипоказана сплошной линией на рис. 4 . По оси ординат отложены координаты тестируемого спектра в двумерной системе координат, соответствующей проекции всей системы в 340 -мерном пространстве. Как правило, спектры предаварийного и исправного оборудования не удается полностью разделить именно эта ситуация и представлена на рис. 4.

При формировании заключения о достоверности дифференциации тестируемого спектра, необходимо формулировать и количественные показатели адекватности. Примем нормальный закон распределения элементов внутри каждого таксона. Тогда вероятность того, что спектр принадлежит к группе аварийных, составит [9] вероятность одновременного наступления двух событий: спектр принадлежит к аварийным, и спектр не принадлежит к исправным:

$$
P=P_{\text {ав }}\left(1-P_{\text {нсп }}\right) \text {, }
$$

где $P_{\text {ав }}$ - вероятность предаварийного состояния агрегата; $P_{\text {нсп }}$ - вероятность бездефектного состояния агрегата.

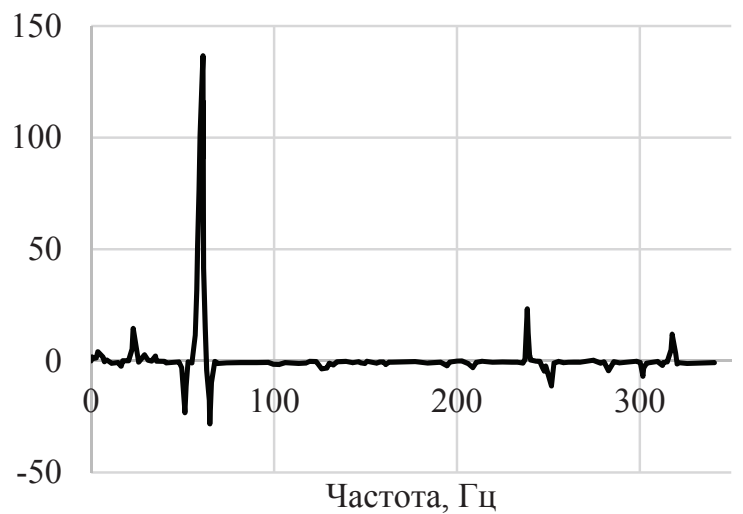

$\sigma$ - третья итерация/third iteration 


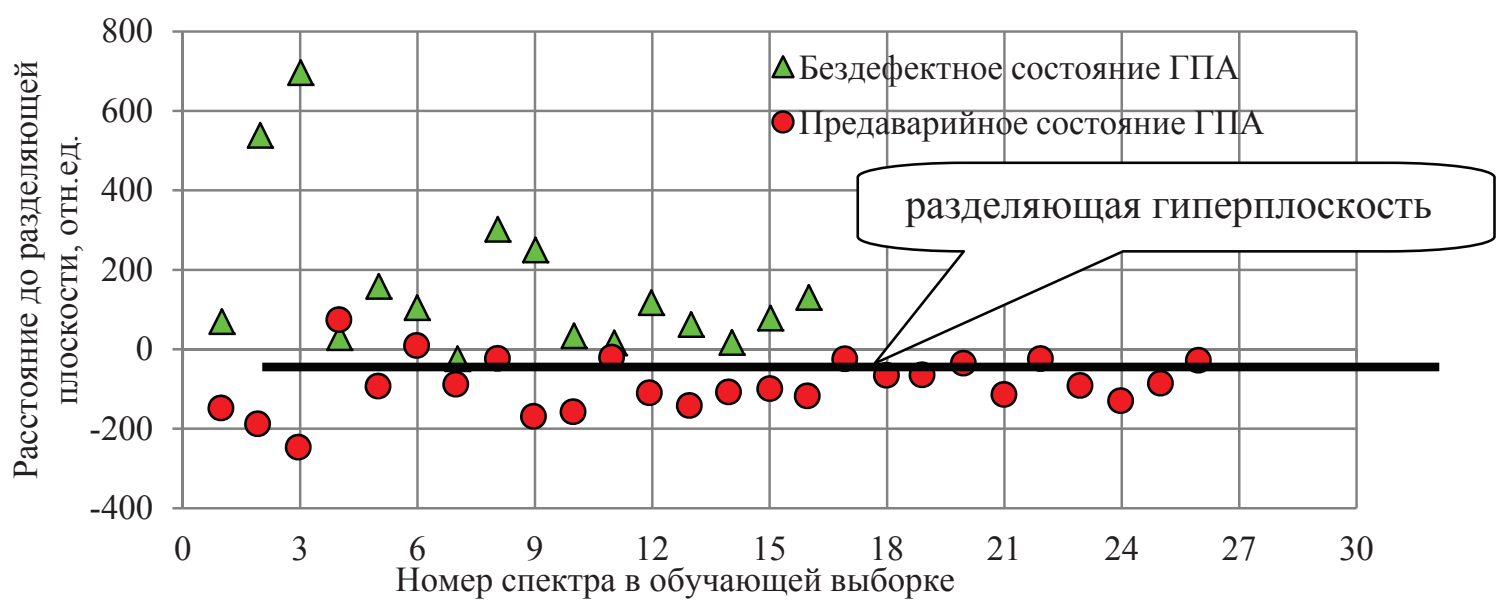

Pис. 4. «Исправные/дефектные» спектры виброскорости таксонов

Fig. 4. «Serviceable/defective» vibration spectra of taxons

Уравнение получено как оценка вероятности одновременного наступления двух независимых событий:

- состояние агрегата предаварийное;

- состояние агрегата небездефектное.

Статистические исследования показателей распределения спектров в каждом таксоне показали, что адекватностьвыводов о механическом состоянии агрегатаопределяется дисперсией и математическим ожиданием распределения спектров в каждом таксоне - минимальное значение дисперсии определяет максимальное значение достоверности заключения. При необходимости дисперсия может быть уменьшена последовательным удалением спектров, по образу отсева грубых погрешностей при анализе результатов эксперимента. Однако при этом неизбежно наступает и снижение достоверности дифференциации таксонов на такую же величину. Поэтому для сохранения достоверности результатов интерпретации спектров рекомендуется удалять лишь некоторое количество отдельных значительно выпавших из таксона точек.

Для оценки качества построенной разделяющей плоскости рекомендуется процедура «скользящий контроль» для расчета вероятности неправильного определения технического состояния агрегата интерпретациейвибросигнала [8].

При компоновке обучающей выборки в ее состав были включены спектры базы данных вибродиагностики, в том числе и максимально близкие к границе раздела таксонов. Такие спектры в дальнейшем и препятствовали однозначному разделению таксонов (рис. 4) и при обучении алгоритма в дальнейшем были исключены из обучающей выборки. По результатам исключения части элементов определяется вероятность ошибочной классификации текущего вибросигнала. Этот показатель рассчитывается по формуле (таблица) и является критерием качества идентифицированной разделяющей гиперплоскости:

$$
P=\frac{m}{l}+\frac{r_{\text {ош }}}{l-m},
$$

где $m$ - количество спектров, удаленных при обучении алгоритма; 1 - первоначальное количество спектров в обучающей выборке сигналов; $r_{\text {ош }}-$ количество неправильно интерпретированных спектров при процедуре «скользящий контроль».

таблица. Определение качества построенной разделяющей поверхности

Table. Determination of the constructed separating surface quality

\begin{tabular}{|c|c|c|c|c|c|c|c|c|c|c|}
\hline \multirow{3}{*}{ 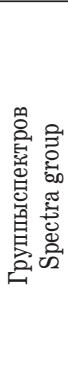 } & \multicolumn{5}{|c|}{$\begin{array}{l}\text { Пообучающейвыборке } \\
\text { By the training sample }\end{array}$} & \multicolumn{5}{|c|}{$\begin{array}{c}\text { При «скользящем контроле» } \\
\text { By the «sliding control» }\end{array}$} \\
\hline & 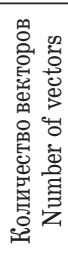 & & 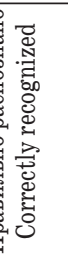 & & 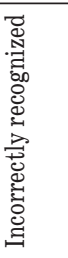 & 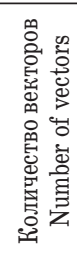 & & 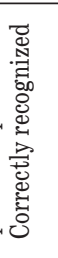 & 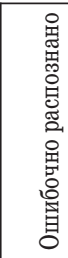 & 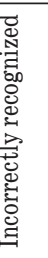 \\
\hline & ед./u & & $(\%)$ & \begin{tabular}{|l|} 
ед. \\
units
\end{tabular} & $(\%)$ & ед./u & & $(\%)$ & \begin{tabular}{|c|} 
ед. \\
units
\end{tabular} & $(\%)$ \\
\hline 1 & 26 & 26 & 100,0 & 0 & 100,0 & 26 & 24 & 92,3 & 2 & 7,7 \\
\hline 2 & 19 & 19 & 100,0 & 0 & 100,0 & 19 & 14 & 73,8 & 5 & 26,2 \\
\hline 3 & 45 & 45 & 100,0 & 0 & 100,0 & 45 & 38 & 84,4 & 7 & 15,5 \\
\hline
\end{tabular}

Принимая во внимание количество элементов, удаленных на стадии обучения алгоритма, вероятность ошибки при оценке механического состояния агрегата по колебаниям корпусов подшипниковых узлов составила $15-30 \%$.

Рассмотренный способ был использован для оценки технического состояния газоперекачивающего оборудования марки ГТК-10. Было установлено, что построение разделяющей геометрической поверхности между таксонами вибросигналов подшипников агрегатов в исправном и предаварийном состоянии могло идентифицировать около $60 \%$ развития дефектов, вызвавших аварийные отказы за четыре года эксплуатации в ПАО «Газпром», не установленные другими способами.

Многократная реализация алгоритма по обработке виброспектров базы данных виброобследований за несколько лет показала, что наиболее высо- 
кая достоверность идентификации класса спектра вибрации достигается при наличии равномерного уровня значимости частот в спектрах виброскорости. Затруднение в классификации вибросигналов вызывает, как правило, широкополосный шум [21].

\section{Заключение}

Для определения механического состояния роторного газотранспортного оборудования интерпретацией виброспектров колебаний подшипниковых узлов турбины низкого давления ГПА рекомендован способ дифференциации таксонов спектров разных состояний плоской поверхностью. Показано, что ошибка интерпретации вёибросигнала уменьшается с ростом объема обучающей выборки, сформированной на основе баз данных виброобследований. Разработка аналитического образа

\section{СПИСОК ЛИТЕРАТУРЫ}

1. Базаров А.А., Данилушкин А.И. Моделирование тепловых и гидравлических процессов в магистральном газопроводе // Известия Томского политехнического университета. Инжиниринг георесурсов. - 2017. - Т. 328. - № 6. - С. 81-90.

2. Байков И.Р., Смородова О.В., Китаев С.В. Оценка параметров надежности агрегатов перекачки магистрального газа // Электронный журнал «Нефтегазовое дело». - 2017. - № 1. C. 95-107. URL: http://ogbus.ru/files/ogbus/issues/1_2017/ ogbus_1_2017_p95-107_BaikovIR_ru.pdf (дата обращения 30.03.2017).

3. Improvement of the composition of detergent solutions for the removal of deposits on the axial-compressor blades of gas-turbine units / I.R. Baikov, A.M. Suleimanov, M.I. Kuznetsova, S.V. Kitaev, Yu.V. Kolotilov // Polymer Science. Series D. - 2018. V. 11. - № 1. - P. 82-85.

4. Шварц А.Ю., Антропов П.Г., Долинина 0.Н. Интеллектуальная система диагностики газоперекачивающих агрегатов компрессорных станций // Новые технологии в газовой промышленности. - Саратов, 16 декабря 2016. - С. 135-145.

5. Байков И.Р., Смородова 0.В., Смородов Е.А. Применение ранговых критериев для вибродиагностики ГПА // Газовая промышленность, - 2000. - № 1. - С. 42-44.

6. Иванов Э.С., Гольянов А.И. Совершенствование процессов эксплуатации газоперекачивающих агрегатов // Нефтегазовое дело. - 2012. - № 1. - С. 42-48.

7. Русов В.А. Диагностика дефектов вращающегося оборудования по вибрационным сигналам. - Пермь: Изд-во «ВиброЦентр», 2012. - 202 с.

8. Vapnik V.N., Chervonenkis A.Y. On the uniform convergence of relative frequencies of events to their probabilities // Measures of Complexity: Festschrift for Alexey Chervonenkis. - New York: Springer International Publ., 2015. - P. 11-30.

9. Chervonenkis A.Y. Problems of machine learning // Lecture Notes in Computer Science. - 2011. - V.6744 LNCS. - P. 21-23.

10. Vetter T., Poggio T. Linear Object Classes and Image Synthesis from a Single Example Image // Transactions on Pattern Analysis and Machine Intelligence. - 1997. - V. 19. - P. 733-742. поверхности разделения таксонов обучающей выборки обеспечивает адекватную оценку состояния ГПА как «исправное/предаварийное» на уровне $60 \%$.

На уровень ошибки классификации спектра виброскорости существенное влияние оказывает временной период развития дефекта агрегата. При развитии дефекта до практически предаварийного состояния достоверность заключения по алгоритму разработанного метода повышается. Снижение адекватности заключения происходит в том случае, когда дефект находится на ранней стадии развития. Подобные результаты имеют место и в случае одновременного развития нескольких дефектов с совмещением спектральных образов. Этот факт обуславливает наличие безошибочно интерпретированных виброспектров на уровне не более $60 \%$.

11. Dai Y., Nakano Y. Recognition of facial images with low resolution using a Hopfield memory model // Pattern Recognition. 1998. - V. 31. - P. 159-167.

12. Химмельблау Д. Анализ процессов статистическими методами. - М.: Изд-во «Мир», 1973. - 957 с.

13. Saaty T.L. Decision making with the analytic hierarchy process // International Journal «Services Sciences». - 2008. - V. 1. № 1. - P. 83-98.

14. Figueira J., Greco S., Ehrgott M. Multiple Criteria Decision Analysis / State of the Art Surveys. - New York: Springer, 2016. P. 27-38.

15. Foresti G.L. Outdoor Scene Classification by a Neural Tree-Based Approach / Pattern Analysis and Applications. - 1999. - V. 2. № 2. - P. 129-142.

16. Temple T.W., Fokz F.L., Jamalallail H.R. System monitors gasturbine maintenance // Oil and Gas Journal. - 1980. - V. 78. № 38. - P. 105-113.

17. Machine learning classification with confidence: application of transductive conformal predictors to MRI-based diagnostic and prognostic markers in depression / I. Nouretdinov, S.G. Costafreda, A. Gammerman, A. Chervonenkis, V. Vovk, V. Vapnik // NeuroImage. - 2011. - V. 56 (2). - P. 809-813.

18. Smorodov E., Deev V. Application of Serial Statistics for Diagnostics of the Oil and Gas Equipment // Journal of Fushun petroleum Institute. - 2000. - № 4. - P. 52-57.

19. Китаев С.В., Иванов Э.С., Галикеев А.Р. Повышение энергоэффективности режимов работы технологического оборудования компрессорных станций магистральных газопроводов. - СПб: Недра, 2016. - 200 с.

20. Bakowski K., Bakowski P. Systemy ochronne w stacjach redukcji gazu // Gaz, Woda i Technika Sanitaria. - 1996. - № 5. P. $167-179$.

21. Байков И.Р., Смородов Е.А., Смородова О.В. Генерация сверхнизких частот при работе газоперекачивающих агрегатов и их влияние на спектр вибрации // Известия высших учебных заведений. Нефть и газ. - 1999. - № 4. - С. 62-67.

Поступила 29.05.2018 г. 


\section{Информация об авторах}

Байков И.Р., доктор технических наук, профессор, заведующий кафедрой промышленной теплоэнергетики Факультета трубопроводного транспорта Уфимского государственного нефтяного технического университета.

Cмородова O.B., кандидат технических наук, доцент кафедры промышленной теплоэнергетики Факультета трубопроводного транспорта Уфимского государственного нефтяного технического университета.

Kuтаев C.B., доктор технических наук, доцент кафедры транспорта и хранения нефти и газа Факультета трубопроводного транспорта Уфимского государственного нефтяного технического университета.

Шамлазов A.M., доктор технических наук, профессор кафедры транспорта и хранения нефти и газа Факультета трубопроводного транспорта Уфимского государственного нефтяного технического университета. 


\title{
SPECIAL METHODS FOR INTERPRETING THE SPEED VIBRATION SPECTRUM FOR DIAGNOSTIC OF GAS-PUMPING UNIT STATE
}

Igor R. Baykov',

pte@rusoil.net

Olga V. Smorodova',

olga_smorodova@mail.ru

\section{Sergei V. Kitaev ${ }^{1}$,}

svkitaev@mail.ru

\author{
Airat M. Shammazov', \\ pte@rusoil.net \\ 1 Ufa State Petroleum Technological University, \\ 1, Kosmonavtov street, Ufa, 450064, Russia.
}

The relevance of the research is caused by the need to develop additional methods for assessing the technical state of gas-pumping units of the main gas pipelines compressor stations. Emergency situations entail consequences of a global nature for the material base and the surrounding environment. The main direction of maintenance of trouble-free compressor stations operation is the maintenance of the technical state of gas-pumping units at the required level.

The main aim of the research is to develop a method for assessing the technical state of gas-pumping units by interpreting the vibration spectrum of oscillations of the bearing housing of the low-pressure turbine of the unit with the formulation of the conclusion "there is a defect/there is no defect».

The objects of research are GTK-10 gas-pumping units operated by PJSC Gazprom. Units of this type provide more than 8 GWt of installed capacity, which is more than $20 \%$ in the country's gas-pumping system. The database for the research is the data of the results of vibration units diagnostics for 4 years.

Methods. To assess the technical state of gas-pumping units based on the interpretation of the vibro-survey spectrum, it is proposed to use the method of constructing a separating surface in a 340-dimensional phase space according to the characteristics of a training sample of vibrospectra. It is established that the reliability of the classification of the spectrum rises with increasing the training sample volume, formed a priori. The greatest effect can be achieved if there are separate strongly marked harmonics in the spectrum.

Results. The authors have constructed a hyperplane to diagnose the technical condition of gas-pumping units of compressor stations of PJSC "Gazprom» by interpreting the spectra of vibration analysis. Taking into account the number of spectra excluded by the separating surface, the probability of erroneous classification of the working vibro-spectra of a gas-pumping unit is set in the range of 0,15-0,30.

\section{Key words:}

Training sample, vibration velocity, separating surface, dimension, algorithm, taxon.

\section{REFERENCES}

1. Bazarov A.A., Danilushkin A.I. Modeling of thermal and hydraulic processes in the main gas pipeline. Bulletin of the Tomsk Polytechnic University. Geo Assets Engineering, 2017, vol. 328, no. 6, pp. 81-90. In Rus.

2. Baikov I.R., Smorodova O.V., Kitaev S.V. Estimation of reliability parameters of aggregates of pumping of the main gas. Electronic scientific journal "Oil and gas business», 2017, no. 1, pp. 95-107. In Rus. Available at: http://ogbus.ru/issues/1_2017/ogbus_1_2017_p95-107_BaikovIR_en.pdf (accessed 30 March 2017).

3. Baikov I.R., Suleimanov A.M., Kuznetsova M.I., Kitaev S.V., KolotilovYu.V. Improvement of the composition of detergent solutions for the removal of deposits on the axial-compressor blades of gas-turbine units. Polymer Science. Series D, 2018, vol. 11, no. 1 , pp. 82-85.

4. Shvarts A.Yu., Antropov P.G., Dolinina 0.N. Intellektualnaya sistema diagnostiki gazoperekachivayushchikh agregatov kompressornykh stantsiy [Intellectual system for diagnostics of gas pumping units of compressor stations]. Novye tekhnologii v gazovoy promyshlennosti [New technologies in gas industry]. Saratov, 16 December 2016. pp. 135-145.

5. Baikov I.R, Smorodova 0.V., Smorodov E.A. Primenenie rangovykh kriteriev dlya vibrodiagnostiki GPA [Application of ran- king criteria for vibrodiagnostics of GPU]. Gazovaya promyshlennost, 2000, no. 1, pp. 42-44.

6. Ivanov E.S., Golyanov A.I. Perfection of operation of gas pumping units. Oil and gas business, 2012, no. 1, pp. 42-48. In Rus.

7. Rusov V.A. Diagnostika defektov vrashchayushchegosya oborudovaniy a po vibratsionnym signalam [Diagnostics of defects of rotating equipment by vibration signals]. Perm, Vibro-Tsentr Publ., 2012. 202 p.

8. Vapnik V.N., Chervonenkis A.Y. On the uniform convergence of relative frequencies of events to their probabilities. Measures of Complexity: Festschrift for Alexey Chervonenkis. New York, Springer International Publ., 2015. pp. 11-30.

9. Chervonenkis A.Y. Problems of machine learning. Lecture Notes in Computer Science, 2011, V.6744 LNCS, pp. 21-23.

10. Vetter T., Poggio T. Linear Object Classes and Image Synthesis from a Single Example Image. Transactions on Pattern Analysis and Machine Intelligence, 1997, vol. 19, pp. 733-742.

11. Dai Y., Nakano Y. Recognition of facial images with low resolution using a Hopfield memory model. Pattern Recognition, 1998, vol. 31, pp. 159-167.

12. Himmelbolou D. Analiz protsessov statisticheskimi metodami [Analysis of processes by statistical methods]. Moscow, Mir Publ., 1973. 957 p.

13. Saaty T.L. Decision making with the analytical hierarchy process. International Journal «Services Sciences», 2008, vol. 1, no. 1, pp. 83-98. 
14. Figueira J., Greco S., Ehrgott M. Multiple Criteria Decision Analysis. State of the Art Surveys. New York, Springer, 2016. pp. 27-38.

15. Foresti G.L. Outdoor Scene Classification by a Neural Tree-Based Approach. Pattern Analysis and Applications, 1999, vol. 2, no. 2, pp. 129-142.

16. Temple T.W., Fokz F.L., Jamalallail H.R. System monitors gasturbine maintenance. Oil and Gas Journal, 1980, vol. 78, no. 38, pp. $105-113$

17. Nouretdinov I., Costafreda S.G., Gammerman A., Chervonenkis A., Vovk V., Vapnik V., Fu C.H.Y. Machine learning classification with confidence: application of transductive conformal predictors to MRI-based diagnostic and prognostic markers in depression. NeuroImage, 2011, vol. 56, pp. 809-813.

18. Smorodov E., Deev V. Application of Serial Statistics for Diagnostics of the Oil and Gas Equipment. Journal of Fushun petroleum Institute, 2000, no. 4, pp. 52-57.
19. Kitaev S.V., Ivanov E.S., Galikeev A.R. Povyshenie energoeffektivnosti rezhimov raboty tekhnologicheskogo oborudovaniya kompressornykh stantsiy magistralnykh gazoprovodov [Increase of energy efficiency of operation modes of technological equipment of compressor stations of main gas pipelines]. St. Petersburg, $\mathrm{Ne}^{-}$ dra Publ., 2016. 200 p.

20. Bakowski K., Bakowski P. Systemy ochronne w stacjach redukcji gazu [Protective systems in gas reduction stations]. Gaz, Woda $i$ Technika Sanitaria, 1996, no. 5, pp. 167-179. In Polish.

21. Baikov I.R., Smorodov E.A., Smorodova O.V. Generatsiya sverkhnizkikh chastot pri rabote gazoperekachivayushchikh agregatov $i$ ikh vliyanie na spektr vibratsii [Generation of ultralow frequencies during operation of gas-pumping aggregates and their influence on the vibration spectrum]. Izvestiya vysshikh uchebnykh zavedeniy. Neft i gaz, 1999, no. 4, pp. 62-67.

Received: 29 May 2018.

\section{Information about the authors}

Igor R. Baykov, Dr. Sc., professor, Ufa State Petroleum Technological University.

Olga V. Smorodova, Cand. Sc., associate professor, Ufa State Petroleum Technological University.

Sergei V. Kitaev, Dr. Sc., associate professor, Ufa State Petroleum Technological University.

Airat M. Shammazov, Dr. Sc., professor, Ufa State Petroleum Technological University. 